INVESTIGACIONES

\title{
APROXIMACIONES A LAS CONCEPCIONES SOBRE DESARROLLO, CONOCIMIENTO Y EDUCACION ABORDADAS EN EL DISCURSO GUBERNAMENTAL
}

\author{
Approaches to the conceptions on development, knowledge and boarded education \\ in the governmental speech
}

\section{Paulina Larrosa}

Universidad Austral de Chile, Facultad de Filosofía y Humanidades, Valdivia, Chile. paulina.larrosa.1@gmail.com

\section{Resumen}

Este estudio indaga las concepciones de Desarrollo, Conocimiento y Educación que proyecta el Gobierno de Chile, en el documento "Hacia una Estrategia Nacional de Innovación para el Desarrollo", elaborado por el Consejo Nacional para la Competitividad, entre los años 2006 y 2008, contemplándose en este análisis los resúmenes ejecutivos de los volúmenes I y II. Se busca, desde una perspectiva crítica y compleja, abordar las dinámicas que emergen y subyacen en dichas concepciones, explorando las contribuciones que el análisis crítico del discurso pueda aportar en la comprensión de las ideologías imperantes en el modelo de desarrollo social vigente en nuestro país. Sostendremos que las concepciones de Desarrollo, Educación y Conocimiento han sido incorporadas en el discurso, por tanto tienen un dominio ideológico latente que arriesga la homogeneización y unidimensionalidad de las estrategias de desarrollo social del país.

Palabras clave: educación, conocimiento, desarrollo, complejidad.

\begin{abstract}
This study investigates the conceptions of development, knowledge and education, that the Government of $\mathrm{C}$ projects spins, in the document "Towards a National Strategy of Innovation for the Development" created by the national advice for the competitiveness, between years 2006 and 2008, being contemplated in this analysis the executive summaries of volumes I and II. One looks for from critical and complex a perspective to approach dynamic which they emerge and they sublie in the these conceptions, being explored contributions that the critical analysis of the speech can contribute in the understanding of the prevailing ideologies in the model of effective social development in our country. We will maintain that the conceptions of development, education and knowledge, have been incorporated in the speech, therefore they have a latent ideological dominion, that risks the homogenización and unidimensionality of the strategies of social development of the country.
\end{abstract}

Key words: education, knowledge, development, complexity. 


\section{INTRODUCCION}

El estudio sistemático de la estructura del discurso ha permitido una aproximación a la comprensión de relatos escritos, públicos y de diversos tipos. La desigualdad y el poder, que conlleva inmersa cierta ideología, pueden ser abordados en general de acuerdo a como estas instancias se evidencian a través del discurso. El Análisis Crítico (ACD) no trata sólo de un análisis descriptivo y analítico, es también un análisis social y político (Van Dijk, 1983). En virtud a lo anterior, se puede comprender la responsabilidad que los investigadores tienen al respecto en la sociedad, permitiendo dilucidar los problemas inherentes a todo acto discursivo.

De acuerdo a los postulados de Foucault (1983), los seres humanos pueden participar en ejercer relaciones discursivas dominantes, asumiendo ideologías y configuraciones de mundo y sociedad, propias de un sistema de dominación. Los estudiantes que han estado expuestos a un sistema particular de poder adquieren de cierta forma elementos cognitivos, sociales, culturales e institucionales propuestos en un sistema educativo que se caracteriza por la replicación y transmisión.

El presente análisis contempla una aproximación a la comprensión, desde una perspectiva lectora y analítica, de discursos obtenidos en base a tres documentos de carácter político-económico, relacionados al concepto de Educación, Desarrollo y Conocimiento, mediante los cuales se pretende visualizar las nociones e ideas que emergen de estos discursos argumentativos y que las sustentan. Dicho análisis será abordado desde la perspectiva crítica de Van Dijk (1980, 1983, 1985, 2000), incorporando, dentro de su línea, los aportes realizados por Bernstein (1993), Fairclough (1995) al estudio de las estructuras discursivas.

\section{MARCO REFERENCIAL}

La poca relevancia que socialmente se le otorga al por qué o para qué educar, está también latente en las políticas públicas, en los discursos educativos y en las agendas globales.

En el discurso cotidiano, en el ámbito relacionado a lo educativo, conceptos económicos como la competencia, eficiencia y satisfacción del consumidor, han desplazado a un segundo plano valores democráticos relacionados al sentido humano como equidad, ciudadanía y solidaridad. Obtener capital humano es la meta más importante del pensamiento educacional contemporáneo. En esta perspectiva economicista, todos los que no son considerados capaces de un alto rendimiento pudiesen quedar relegados a funciones que perpetúan la discriminación y el abuso de poder.

Algunos estudios acuerdan en proponer que la complejidad del mundo en que hoy se vive, ha puesto en jaque las instituciones y organizaciones sociales, trastocándose los saberes, ideas y valores, fundamentos claves de la educación correspondiente a cierta cultura (Morin y Kern, 1993; Giddens, 1997; Castells, 1997).

Existen procesos que, aunque silenciosos, se encuentran cotidianamente invadiendo los fundamentos, principios y prácticas en torno a lo educativo, que merecen un análisis crítico que evidencie las correspondientes esferas de poder que se encuentran ocultas en las prácticas y discursos. Como lo señala Brunner (2000), son muchos los factores 
que afectan e inciden poderosamente en una cultura, en la demografía, en la actitud de las familias, en las condiciones económicas y políticas. Las ideas que existen sobre la forma de educar están relacionadas, como lo advierte el autor, a factores internos, como también externos al propio sistema educacional, a la organización y la administración de las instituciones relacionadas a la enseñanza.

Según Coraggio (1995), el análisis económico se ha transformado en la metodología central para el diseño de las políticas educativas; en tal sentido, las tendencias al desarrollo y crecimiento advierten la adecuación y adaptación de los ámbitos relacionados a lo educativo. Por ello, sugiere que la acción renovadora no depende únicamente de los componentes intrínsecos, como gestores de un cambio global, sino más bien surge como un ajuste a otros problemas sociales que, aunque igualmente importantes, podrían ser el resultado de los cambios fundamentales proyectados holográficamente desde el ámbito educativo. De esta forma, si los cimientos no son reconfigurados primero, difícilmente habrá transformaciones epistemológicas en educación.

Considerando los antecedentes anteriores, el ADC constituye una herramienta importante para el análisis social, político y cultural, comprendiendo que todo fenómeno educativo participa en la construcción de la identidad cultural, pudiendo lograr entrever las ideologías y relaciones de poder en la construcción de los discursos, de acuerdo a las concepciones que los mismos actores proponen mediante su acción discursiva. Esta herramienta, puede ser concebida como una metodología o bien como una teoría, ofreciendo como perspectiva de investigación la posibilidad de esclarecer determinados aspectos en las concepciones sobre Educación, Desarrollo y Conocimiento, en determinados momentos históricos.

De acuerdo a Fairclough (1995) el ACD se concibe como el análisis de las relaciones dialécticas entre la semiosis, en la cual se encuentra incluido el lenguaje y otros elementos de las prácticas sociales. Por otra parte, Van Dijk (2000) propone que el ACD no corresponde ni a un método ni una teoría, pudiendo comprender y abarcar cualquier enfoque en las ciencias humanas y las ciencias sociales, advirtiendo la necesidad de ampliar el ACD y vincularlo con otros enfoques.

La hegemonía material y simbólica que impera a nivel social opera a su vez política y pedagógicamente redefiniendo la noción de desarrollo. El indagar en los discursos educacionales en relación al desarrollo permite comprender el impacto en la construcción de una sociedad más justa y democrática. Es importante considerar que las políticas públicas en relación al ámbito educativo se fundamentan y establecen de acuerdo a las luchas de sentido que presentan los actores involucrados en este proceso. En consideración a estas concepciones y percepciones, se satisfacen las necesidades orientadas a propuestas concretas de mejora.

La presente exploración permite indagar en las nociones sobre Desarrollo, Conocimiento y Educación, percibiendo cómo los continuos discursos educativos, que se han repetido durante la formación, intervienen en las concepciones personales y prácticas educativas, contribuyendo a su vez en la construcción de identidades locales, nacionales y de imaginarios sociales y culturales. Bernstein (1993) desarrolla un planteamiento de un análisis sistemático del discurso como de la práctica pedagógica, caracterizando y distinguiendo la forma especializada de comunicación que tiene lugar en los discursos pedagógicos, desarrollando prácticas educativas basadas en las clases sociales, lo que puede evidenciar en ciertos argumentos relacionados a lo educativo, el manejo político del poder. 
En vista que el discurso será considerado en este estudio como texto escrito, es relevante mencionar la manera en que es abordado el lenguaje, el cual toma forma en la búsqueda y configuración de sentidos como tarea central de todo el proceso dialéctico. En consecuencia, no se puede dejar de considerar la ambigüedad y polisemia de éste, originada en la perspectiva estructural o sintáctica, mas a ella debiera estar asociada la dimensión comunicacional que sobrepasa las perspectivas de órdenes lineales (von Foerster, 1996). En este sentido, la noción de lenguaje es abordada desde una concepción compleja comprendiendo su dimensión semántica, comunicacional y generadora de espacios de sentido, "haciendo referencia a nuestra comprensión genérica -nuestra interpretación- de lo que significa ser humano" (Echeverría, 1994; 28).

\section{METODOLOGIA}

El modelo metodológico que se ha considerado para nuestra investigación se enmarca dentro del posicionamiento de Van Dijk (2000), de acuerdo al modo en que ejercen el poder algunos hablantes o escritores en, o por medio de su discurso, considerando para el análisis los lineamientos aportados por el autor como: el análisis de macroestructuras semánticas, análisis de las formas o formatos del discurso global y local, análisis de las específicas realizaciones lingüísticas, como del contexto.

Bajo esta perspectiva metodológica se considerará el discurso como texto, como lo propone Fairclough (1995), en tanto producto oral o escrito de una producción discursiva, entendido como práctica que se inserta en una situación social determinada. El discurso puede, como práctica social, estructurar áreas de conocimiento, expresar o reflejar entidades, relaciones, como también constituirlas y conformarlas. Así, el análisis en este trabajo se realizará de forma autónoma y semántica, incluyendo el tema y el contenido, que presentan relación con los procesos inherentes a la creación del texto.

Las nociones de implicatura y presuposición constituyen elementos fundamentales para el análisis del discurso, debido a que la mayor parte de las concepciones ideológicas como la dominación y abuso de poder se encuentran implícitas en los distintos niveles del mismo. Mediante la implicatura, el receptor deduce información no explicitada en el enunciado por el emisor, analizando no sólo lo explicitado o emitido, sino también aquello que, aunque no se expresa verbalmente, se encuentra implícito en el enunciado. Por otra parte, en la presuposición, el o los hablantes suponen lo que el o los oyentes conocen sobre un determinado tema; es una noción ligada a la verdad, la que es una condición del acto de hablar para que sea verdad (Ferrer 1996). "La presuposición, en cuanto acto semántico de índole mental, opera en el terreno de lo conocido, de tal forma que a través de ella el emisor imagina las cosas que el receptor ya sabe" (Van Dijk, 1980: 313).

A continuación se pretende vincular la técnica de análisis que se extrae de los planteamientos de Van Dijk para el ACD con las redes semánticas, comprendiendo que éstas podrían actuar distinguiendo las proposiciones de un discurso en determinadas circunstancias. El proceso dinámico de este estudio permite un enfoque y diseño con una estrategia metodológica de investigación, que apunta a la crítica del discurso en cuanto sistema dinámico. El carácter dialógico que ello supone viene dado, en una parte, por la interacción producida en los procesos de distinción en la que participa el propio investigador (elementos implícitos) y, por otra, la dialéctica de la relación entre sujeto y objeto a través de la comunicación que establece él o los hablantes en el discurso (elementos explícitos) en un contexto histórico social específico en relación a la concepción de Desarrollo, Educación y Conocimiento. 
La técnica de análisis se basa en el cartografiado computacional que grafica las principales ideas que se distinguen del plano argumentativo, organizándolo en base a conceptos y conexiones. Este tipo de modelamiento de ideas, según (Lavanderos y Malpartida, 2000), da cuenta del marco o paradigma, desde donde el observador construye su observación. De esta forma, los conceptos quedan definidos como las distinciones a partir de un tema en particular y el paradigma o pensamiento como la red de asociaciones entre las distinciones. Las distinciones utilizadas en la presente investigación se diferencian en asociativas, que corresponden a conceptos que interponen parte de sus significados con otros y causales, que corresponden a conceptos en los cuales la presencia de uno afecta al otro. De tal manera el modelamiento permitirá distinguir las principales ideas en las cuales se fundamenta el discurso y los pilares en los cuales se sostienen y emergen las concepciones de Desarrollo, Conocimiento y Educación.

Gracias a este análisis, es posible encontrar algunas distinciones del plano argumental que centralizan y pautan la relación de ideas, permitiendo identificar las nociones asociadas a los conceptos antes mencionados, que surgen en el entramado configuracional, como las ideas centralizadoras que guían y median el contexto general en el que se expanden las nociones de Desarrollo, Conocimiento y Educación. Para este estudio la secuencia de análisis será la siguiente:

1. Se visualiza en primera instancia la argumentación de los planos observacionales para identificar las principales distinciones, indagando y enunciando los atractores o proposiciones, que son las ideas principales que pautan el discurso, como asimismo el cartografiado de la configuración de distinciones, en la cual ellos son definidos y tienen sentido. Esto, dando énfasis en la organización y relación de las ideas que emergen. Es importante señalar que el cartografiado corresponde a una aproximación momentánea y particular de las ideas que expresan los informantes, en este caso las personas participantes en el discurso escrito, el cual debe ser entendido en el carácter dinámico que adquiere en el proceso de configuración de sentido. El cartografiado es representado mediante una red de semiosis, que integra los principales atractores, las ideas o distinciones relacionadas a él y las conexiones que se establecen, de acuerdo al grado de causalidad y asociatividad.

\section{Figura 1}

Esquema que representa un atractor dentro de la red de semiosis

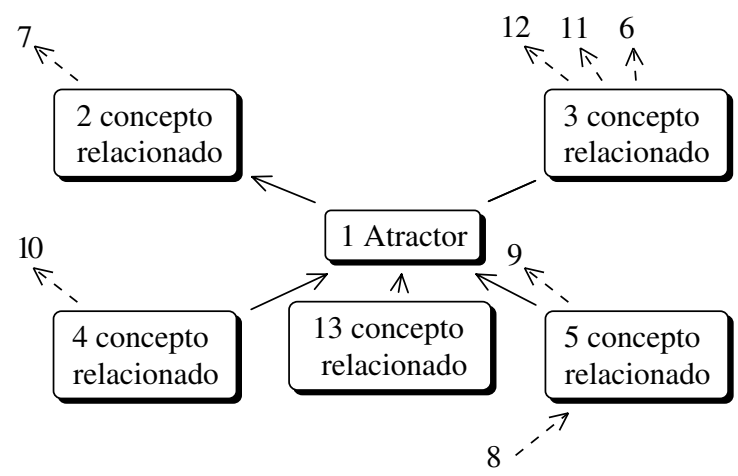


2. Una vez obtenidas las principales proposiciones o ideas centralizadoras a partir del punto anterior, se seguirán las normas de coherencia, permitiéndose de esta forma que en el análisis del texto se establezcan las relaciones manifiestas entre las diversas proposiciones como, a su vez, la aproximación a los procesos cognitivos ligados a la asignación de significado de un discurso, que corresponde a la coherencia global representada por las macroestructuras y superestructuras. De acuerdo a Van Dijk (1985:115), las macroestructuras corresponden a la..."reconstrucción teórica de nociones equivalentes a las del tema, asunto o tópico de un discurso". Por esto, para la comprensión crítica del texto se seguirán las siguientes pautas de análisis:

\section{Figura 2}

Representación de las normas de coherencia que se siguen para el análisis (Adaptación de la propuesta de Van Dijk, 1983)

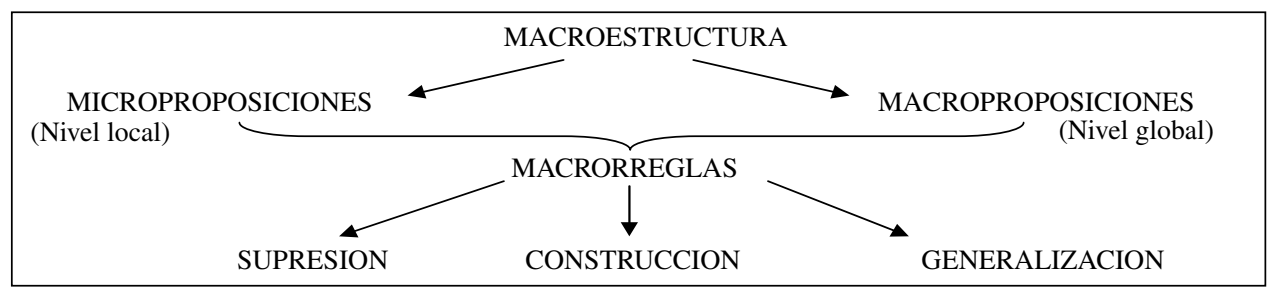

Se propone, entonces, que las macroproposiciones podrían ser comprendidas como el análisis del contexto global que emerge de la configuración de ideas (asociaciones que se establecen en el análisis), no explicitado en el contenido propio del texto y las microproposiciones serían comprendidas como las ideas explicitadas en el discurso que lo pautan y dirigen. Las macrorreglas nos permitirán reformular el texto realizando operaciones de supresión, borrando o eliminando información secundaria, manteniendo la información relevante, por otra parte integrando y construyendo a partir de la información de algunos enunciados otros que los contengan.

\section{RESULTADO ANALISIS}

\subsection{TOTAL DE CONCEPTOS INCLUIDOS EN LA RED SEMIOSIS}

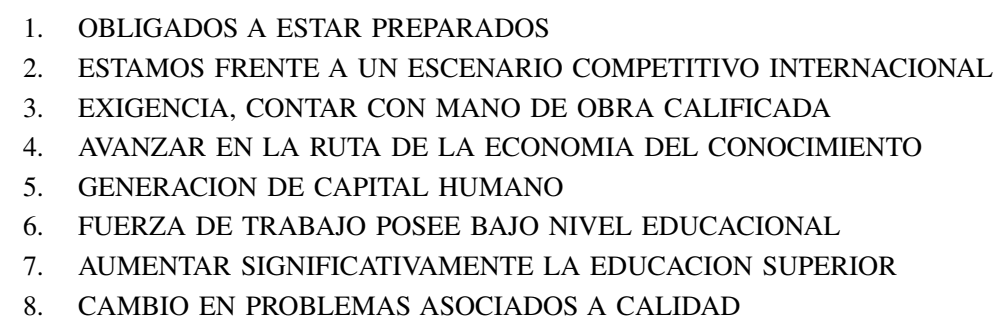




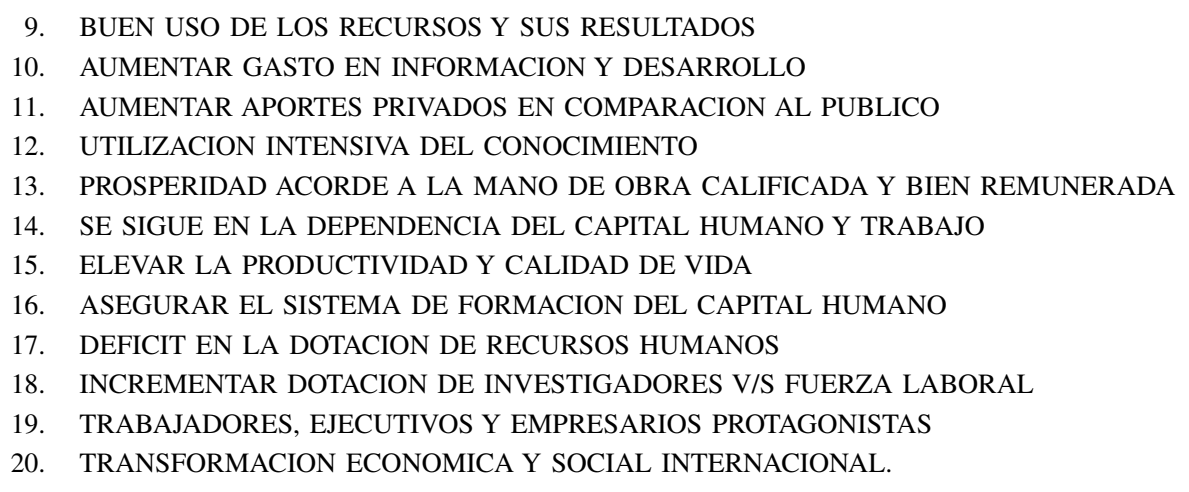

\subsection{RED DE SEMIOSIS}

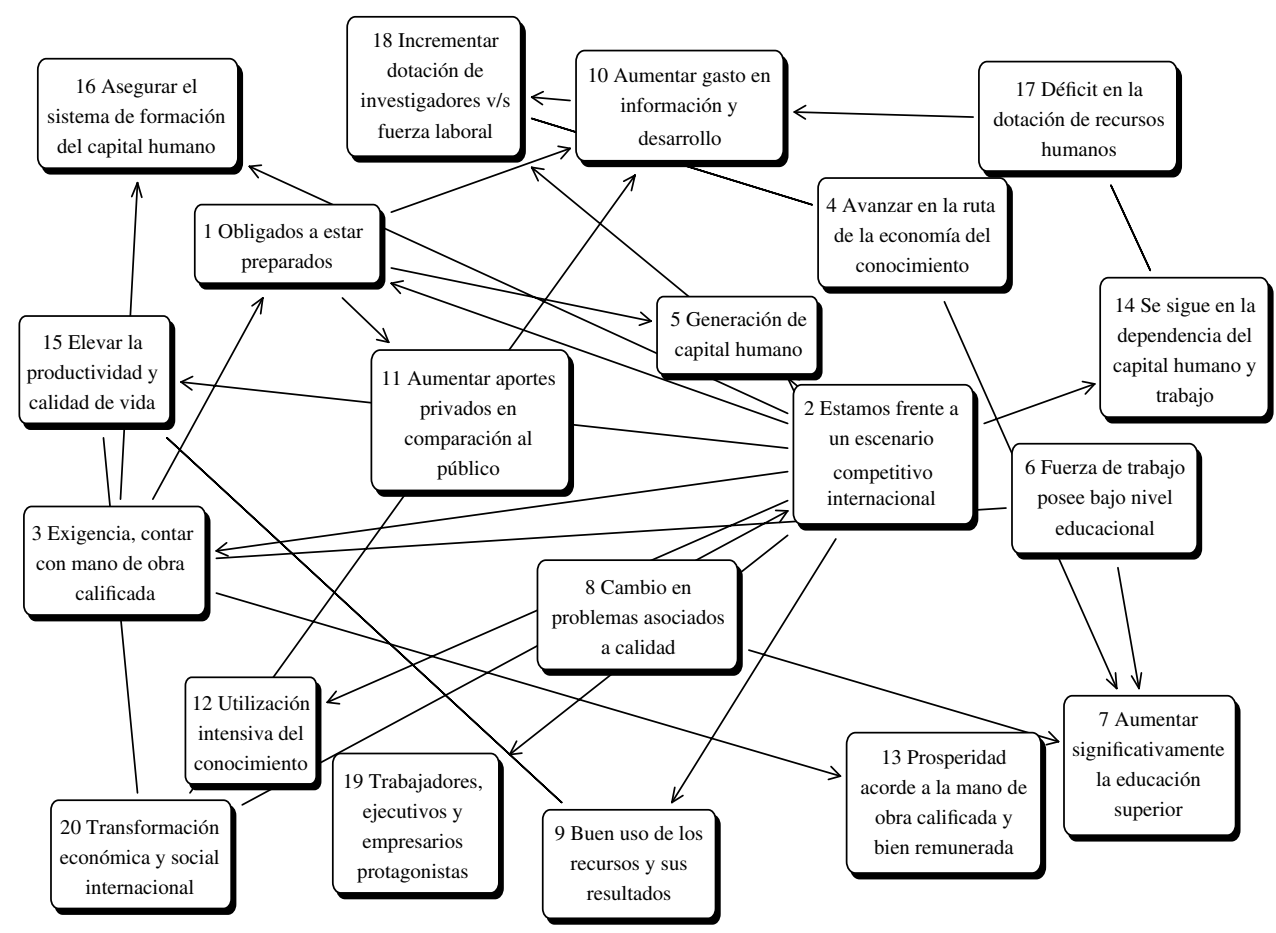

\subsection{ANALISIS DE DOMINIO}

\begin{tabular}{|c|l|}
\hline 11. LINKS ALREDEDOR & 2. ESTAMOS FRENTE A UN ESCENARIO COMPETITIVO INTERNACIONAL \\
\hline 5. LINKS ALREDEDOR & 1. OBLIGADOS A ESTAR PREPARADOS \\
\hline 4. LINKS ALREDEDOR & $\begin{array}{l}\text { 3. EXIGENCIA, CONTAR CON MANO DE OBRA CALIFICADA } \\
\text { 10. AUMENTAR GASTO EN INFORMACION Y DESARROLLO }\end{array}$ \\
\hline
\end{tabular}




\begin{tabular}{|c|c|}
\hline 3. LINKS ALREDEDOR & 7. AUMENTAR SIGNIFICATIVAMENTE LA EDUCACION SUPERIOR \\
\hline 2. LINKS ALREDEDOR & $\begin{array}{l}5 \text { GENERACION DE CAPITAL HUMANO } \\
\text { 16. ASEGURAR EL SISTEMA DE FORMACION DEL CAPITAL HUMANO } \\
\text { 18. INCREMENTAR DOTACION DE INVESTIGADORES V/S FUERZA LABORAL } \\
\text { 20. TRANSFORMACION ECONOMICA Y SOCIAL INTERNACIONAL }\end{array}$ \\
\hline 1. LINK ALREDEDOR & $\begin{array}{l}\text { 4. AVANZAR EN LA RUTA DE LA ECONOMIA DEL CONOCIMIENTO } \\
\text { 6. FUERZA DE TRABAJO POSEE BAJO NIVEL EDUCACIONAL } \\
\text { 8. CAMBIO EN PROBLEMAS ASOCIADOS A CALIDAD } \\
\text { 9. BUEN USO DE LOS RECURSOS Y SUS RESULTADOS } \\
\text { 11. AUMENTAR APORTES PRIVADOS EN COMPARACION AL PUBLICO } \\
\text { 12. UTILIZACION INTENSIVA DEL CONOCIMIENTO } \\
\text { 13. PROSPERIDAD ACORDE A LA MANO DE OBRA CALIFICADA Y BIEN REMUNERADA } \\
\text { 14. SE SIGUE EN LA DEPENDENCIA DEL CAPITAL HUMANO Y TRABAJO } \\
\text { 15. ELEVAR LA PRODUCTIVIDAD Y CALIDAD DE VIDA } \\
\text { 17. DEFICIT EN LA DOTACION DE RECURSOS HUMANOS } \\
\text { 19. TRABAJADORES, EJECUTIVOS Y EMPRESARIOS PROTAGONISTAS }\end{array}$ \\
\hline
\end{tabular}

\subsection{ANALISIS DE CENTRALIDAD}

Primeros 3 atractores son los más centrales en el proceso

2. ESTAMOS FRENTE A UN ESCENARIO COMPETITIVO INTERNACIONAL

3. EXIGENCIA, CONTAR CON MANO DE OBRA CALIFICADA

18. INCREMENTAR DOTACION DE INVESTIGADORES V/S FUERZA LABORAL

1. OBLIGADOS A ESTAR PREPARADOS

20. TRANSFORMACION ECONOMICA Y SOCIAL INTERNACIONAL

10. AUMENTAR GASTO EN INFORMACION Y DESARROLLO

19. TRABAJADORES, EJECUTIVOS Y EMPRESARIOS PROTAGONISTAS

16. ASEGURAR EL SISTEMA DE FORMACION DEL CAPITAL HUMANO

15. ELEVAR LA PRODUCTIVIDAD Y CALIDAD DE VIDA

14. SE SIGUE EN LA DEPENDENCIA DEL CAPITAL HUMANO Y TRABAJO

12. UTILIZACION INTENSIVA DEL CONOCIMIENTO

9. BUEN USO DE LOS RECURSOS Y SUS RESULTADOS

6. FUERZA DE TRABAJO POSEE BAJO NIVEL EDUCACIONAL

5. GENERACION DE CAPITAL HUMANO

17. DEFICIT EN LA DOTACION DE RECURSOS HUMANOS

4. AVANZAR EN LA RUTA DE LA ECONOMIA DEL CONOCIMIENTO

13. PROSPERIDAD ACORDE A LA MANO DE OBRA CALIFICADA Y BIEN REMUNERADA

11. AUMENTAR APORTES PRIVADOS EN COMPARACION AL PUBLICO

7. AUMENTAR SIGNIFICATIVAMENTE LA EDUCACION SUPERIOR

8. CAMBIO EN PROBLEMAS ASOCIADOS A CALIDAD 


\subsection{PRINCIPALES ATRACTORES}

Atractor A (concepto 2: Estamos frente a un escenario competitivo internacional)

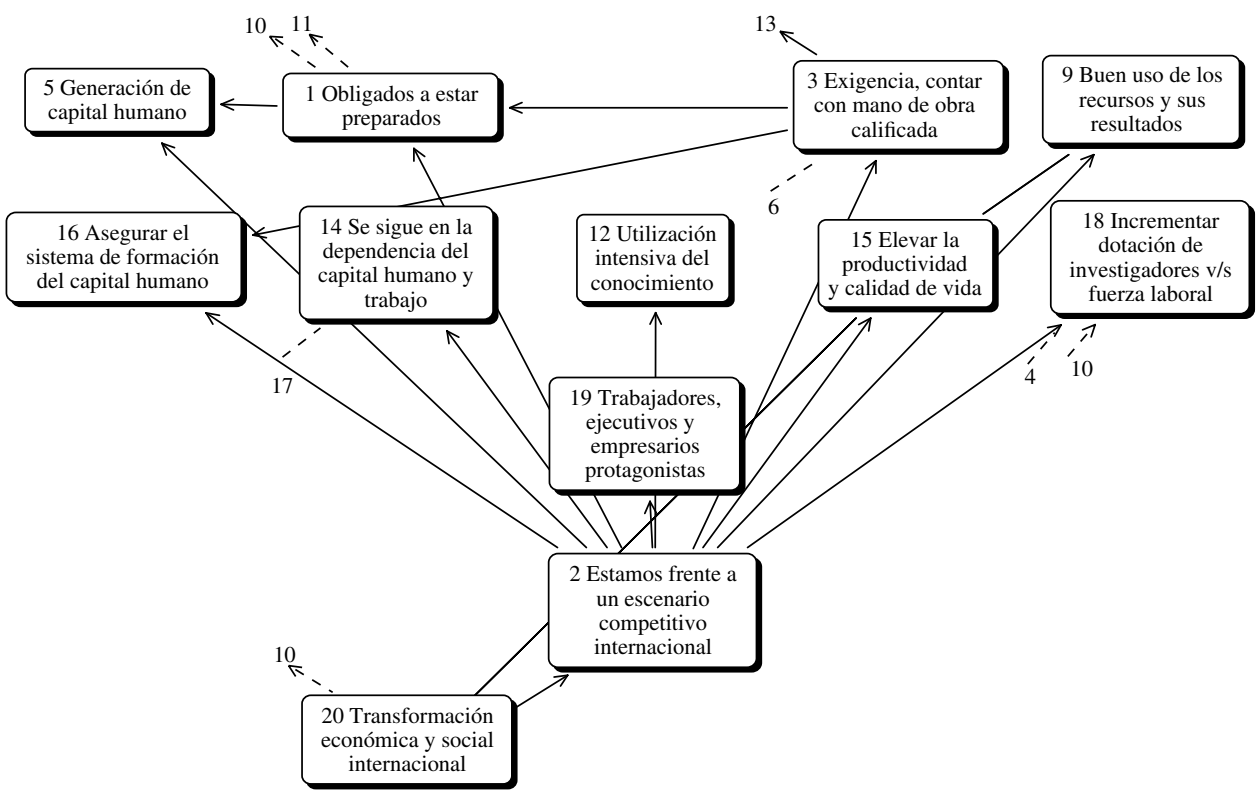

Atractor B (concepto 3: Exigencia: contar con mano de obra calificada)

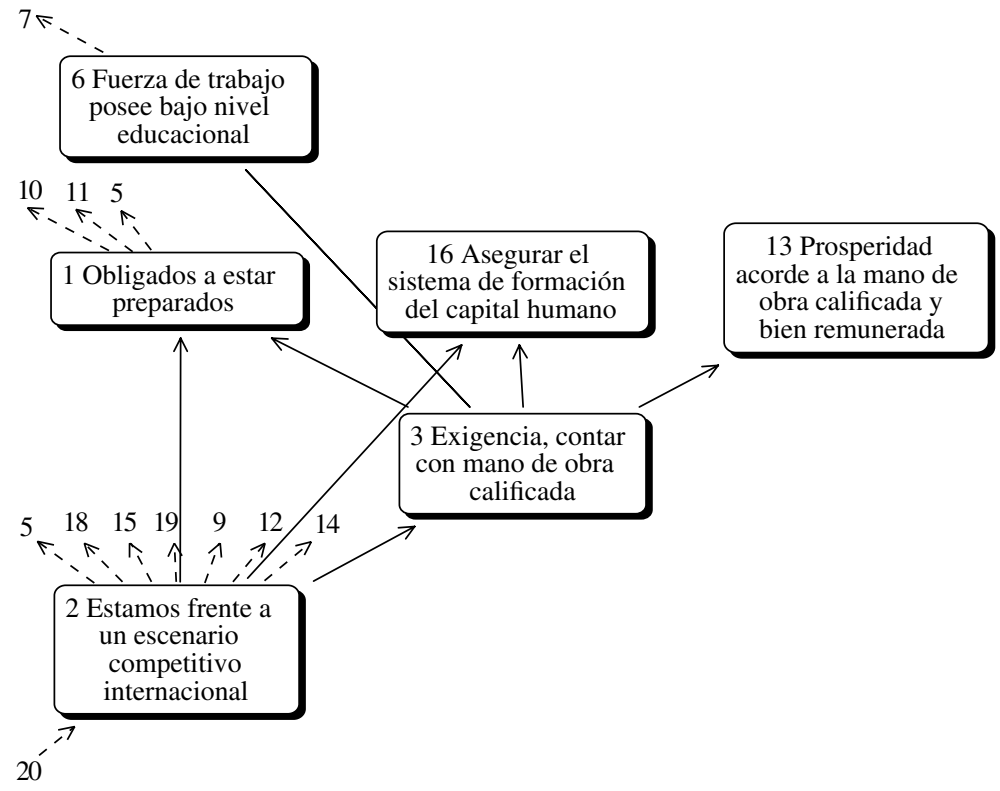


Atractor C (concepto 18: Incrementar dotación de profesores v/s fuerza laboral)

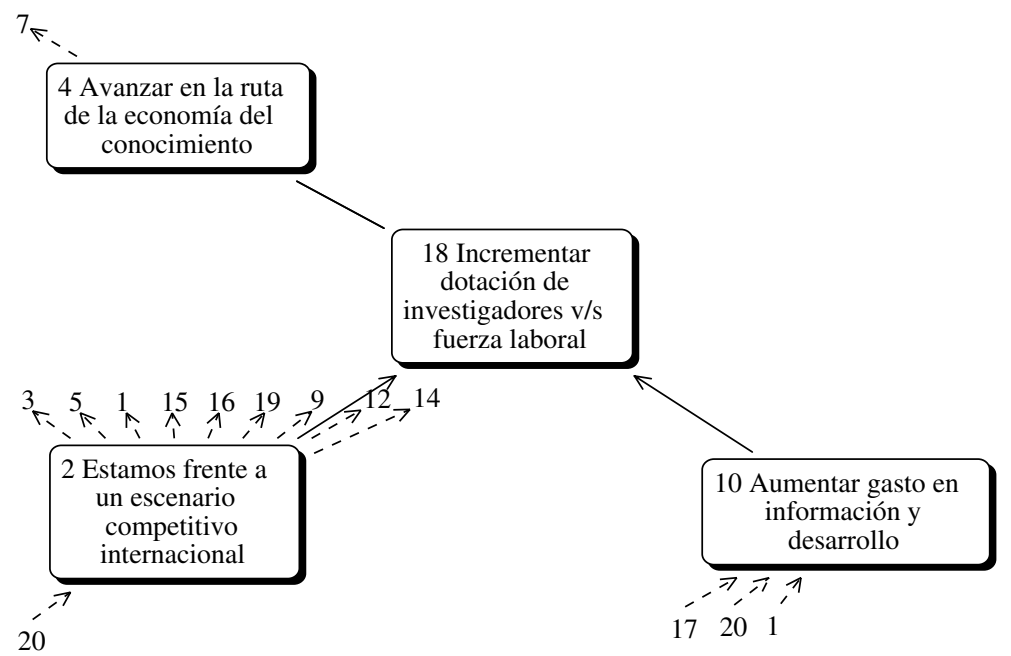

\section{ANALISIS CRITICO}

A continuación se interpreta y da sentido a los principales atractores que se aprecian en el documento en torno al Desarrollo, Conocimiento y Educación.

\subsection{IDEOLOGIA Y EDUCACION DESDE EL DISCURSO}

Al analizarse la importancia del planteamiento de la propuesta educativa, desde una perspectiva discursiva y las implicaciones que esto presenta en los retos que la sociedad exige, se puede vislumbrar y abordar de manera compleja y global el fenómeno, asociando las ideas que los hablantes proponen en su discurso.

$\mathrm{Si}$ analizamos de manera conjunta los tres atractores más importantes que dan lugar al discurso, dentro del plano de distinciones, tenemos que las ideas: estamos frente a un escenario competitivo internacional (2), exigencia, contar con mano de obra calificada (3), incrementar dotación de investigadores v/s fuerza laboral (18), hacen énfasis en una concepción unidimensional basada en consideraciones de progreso económico y competitivo.

El concepto de formación, como una temática que pudiera estar asociada a la noción educativa aparece en la posición 16, presentada como: asegurar el sistema de formación del capital humano. Esta idea evidencia la pobre relevancia que presenta la formación en términos de desarrollo social, centrada en la idea de capital humano. Los sujetos son reducidos, mediante esta conceptualización a meros agentes que necesitan preparación y capacitación para enfrentar los nuevos desafíos, no siendo vistos ellos mismos agentes forjadores de transformación social.

Es necesario, frente a este panorama, que la educación reconozca su carácter generativo y no sólo adaptativo a la altura de estos tiempos. Según Carrizales (1991) existe una 
hiperactualización ${ }^{1}$ que trata de implementar vertiginosas innovaciones que alcanzan el ámbito educativo, como también las creencias, valoraciones, capacidades y expectativas. De esta forma se adoptan urgentemente modelos que no han permitido la reflexión necesaria, siendo el objetivo primordial el no quedarse atrás, esto es, no quedarse fuera de esta competencia a la cual son arrastrados países como el nuestro, en donde la globalización es asumida como una forma de agenciarse a una noción de sociedad del conocimiento.

De esta manera, las transformaciones en casi todas las dimensiones sociales y esenciales de una cultura se ven alteradas, anticipando que también la educación verá alterado su curso histórico. La educación no permanece estable, se ve forzada a adaptarse a las nuevas circunstancias, siempre primando las características de orden económico por sobre un posicionamiento epistemológico crítico y autónomo desde los sistemas educativos. Los cambios son adaptativos, se acomodan al ritmo variante de otros acontecimientos que, aunque no menos relevantes, la relegan a ciertos niveles de desvalorización y adaptación.

Existen importantes aspectos a considerar, que según Morin (1999), impiden y bloquean un cambio en las coherencias paradigmáticas de la educación y, con ello, una reforma no sólo a nivel de las instituciones sino también del pensamiento. La relación entre sociedad y escuela no debiese ser igualada a la de un sistema espejo, sino más bien a la de un holograma que representa en sus características más singulares y fundamentales la presencia de la sociedad por completo. Las tendencias recurrentes de la educación a la adaptación y adecuación de los cambios sociales hacen que ésta sea percibida como resultado y no como proceso, debiendo superar esta visualización para entender que la educación produce la sociedad que produce la educación.

La preocupación que muestra el discurso en dar relevancia a la educación como fuente importante de cambio lleva a cuestionarse cuál será el real interés que existe en distinguir al ciudadano como: fuerza de trabajo, que posee bajo nivel educacional (concepto 6). Se dilucida entonces que es necesario aumentar el nivel educacional de las personas, necesariamente porque aquello debilita la fuerza de trabajo y, al parecer, como lo explicitan las otras ideas, no se puede estar a un nivel competitivo internacional (concepto 2). Esta idea representa un nodo crítico y fundamental dentro de la argumentación, apareciendo como concepto central en la configuración del discurso para la innovación y desarrollo del país.

Desde los planteamientos de Oliva (2007), los programas de acción destinados a la transformación educativa se desarrollan en el contexto de una transversal reivindicación de los sistemas educativos a nivel planetario, estrechamente relacionada con importantes transformaciones en las relaciones sociales, económicas y culturales. De acuerdo a estos fenómenos, se podría hablar de un tránsito desde una sociedad industrial a economías fuertemente mediadas por el conocimiento, procesos que han venido a situar a los sistemas educativos como agentes principales de cambio a nivel planetario. No obstante, el aspecto lineal en el ámbito social propuesto por Chomsky y Dieterich (1999), en que la economía da lugar al trabajo y posteriormente a la educación, mantiene su hegemonía

Concepto que Carrizales (199: 3-4) propone para explicar el aceleramiento de la velocidad y la obsesión pedagógica por sintonizar con la actualización de los signos contemporáneos, pretendiendo a partir de ella determinar el atraso o progreso, el fracaso o el éxito, la pobreza o la riqueza. 
en la esfera social y cultural, perpetuando el determinismo y la linealidad que engendran los sistemas carentes de una visión multidimensional.

Según Martínez (2001) los problemas que debe enfrentar la educación en el escenario global, se encuentran asociados al paradigma productivo imperante que pretende abrirse a las nociones de desarrollo, conocimiento, progreso técnico, innovación y creatividad. Bajo esta perspectiva, los discursos gubernamentales dirigen sus planteamientos y posibles soluciones a una insistencia y cada vez más recurrente énfasis en los aspectos relacionados a la inversión, en términos económicos, siendo que el problema es más bien epistemológico. Como lo advierte Morin (1999), no se pueden reformar las instituciones sin reformar las mentes y no se pueden reformar las mentes sin reformar las instituciones.

Es importante que en toda esta dinámica surja el pensamiento crítico y expansivo, en el cual los actores logren ser partícipes de los acontecimientos que ellos mismos están viviendo, forjando y produciendo individuos conscientes, personalidades con carácter decisivo, para no ser llevados por corrientes que desvaloricen la existencia humana y conviertan a los hombres en meras máquinas capacitadas, para cumplir mejor su labor. Por esto, el formar una conciencia amplia y abierta a la diversidad logra ser importante $\mathrm{y}$ fundamental en el proceso.

Cuando se considera que el discurso permea los procesos educativos y toda acción pedagógica, éste puede otorgar la posibilidad de educar "sujetos discursivos intencionales y autónomos con poder de interlocución, que tengan acceso a la experiencia del discurso argumentado y a la posibilidad de análisis para intervenir en la toma de decisiones", (Martínez 2001:141). De esta manera, las lógicas que generan ciertos discursos pueden ser cuestionadas en un marco de crítica y reflexión, sin caer en el adaptacionismo inconciente de decisiones tomadas unilateral y unidimensionalmente.

\subsection{NOCION DE DESARROLLO}

Las altas expectativas de desarrollo manifestadas en el documento advierten ciertas necesidades explicitadas en las nociones de: exigencia, contar con mano de obra calificada (concepto 3), incrementar dotación de investigadores v/s fuerza laboral (concepto 18), aumentar gasto en información y desarrollo (concepto 10), buen uso de los recursos y sus resultados (9), prosperidad acorde a la mano de obra calificada y bien remunerada (13), aumentar aportes privados en comparación al público (11).

La competencia global e internacional hacen que el desarrollo se sustente en aspectos que permiten la eficiencia en términos productivos, la mano de obra calificada sólo será posible si el país se rige a niveles de estándares internacionales que, tal vez, nada tienen que ver con las realidades particulares de las sociedades latinoamericanas. Por otra parte, la concepción deshumanizada que pretende dar triunfo a los mercados en las esferas globales, compitiendo al mismo nivel que las consideradas grandes naciones, en donde sólo se contempla el desarrollo en función de la calidad de vida en términos económicos. En ello, no dan cuenta de las carencias que en otros ámbitos se puedan observar, debilitando la idea de sociedades colaborativas, dando lugar a sociedades productivas que pierden el sentido de desarrollo multidimensional de un país.

Como lo menciona Morin (2002: 393) "Se ha construido la idea de desarrollo sobre la base de un mito humanístico/racionalista, unidimensional y pobre del hombre y sobre la base de una idea mecanicista/economicista sorprendentemente limitada de la sociedad", 
el concepto de desarrollo latente en los discursos no deja de ser en palabras del mismo autor, simplificador, mutilante, mecánico, lineal, racionalizador y eufórico, los índices de desarrollo permanentemente son representados mediante gráficas económicas que delimitan la condición humana y la restringen a aspectos triviales, siendo claramente el único índice medible de desarrollo el propio crecimiento industrial. Desde esta base, se dejan relegadas las opciones de desarrollo humano que trascienden estas esferas; el ser humano por tanto tiene cabida en este espacio social como constructor de una lógica mecánica y productiva que invalida su opción reflexiva, crítica y participativa.

Falta incluir al hombre o mujer, quienes han sido aminorados por la visualización que se presenta de su condición, es más, abordar la noción de desarrollo es abordar el autodesarrollo. Auto=hombre (sociedad, individuo, el desarrollo debe, por tanto, concebirse en forma ilimitadamente recursiva en la que el desarrollo se convierte a la vez en el medio y el fin del sistema autoorganizador (sociedad-individuo) (Morin 2002). Esta visión permite que a la hora de incluir al ser humano en los procesos productivos, no se olvide que lo fundamental e impostergable es el autodesarrollo, la clave para una metamorfosis social efectiva.

La información aparece como importante dentro del plano argumentativo, para lograr mejoras en vistas a una sociedad más desarrollada. Algunas interpretaciones y concepciones acerca de la información nos hablan de que es aquella que sería percibida por el sistema cognitivo y luego representada en forma de conceptos que son manifestados a través del lenguaje (Torres 1999). De acuerdo a lo abordado por este autor, la información no siempre es la misma, ya que cada individuo tiene la capacidad de reconfigurar el mundo de distinciones que le son posibles o que bien, le son significativas; de allí la importancia en que la información otorgada sea coherente, pertinente y con una orientación adecuada para promover la mejor utilización de esta información, en virtud del sentido que comprende el progreso y desarrollo de un país.

La información es importante para generar una conciencia local y planetaria que permita entender los propósitos particulares, como aquellos generales que pautan las lógicas de desarrollo. Esta conciencia, como lo advierte Morin (2002), está lejos de comprender la gran carencia de todo progreso político y social en la idea de desarrollo, ya que no existe un modelo todavía que logre dar fundamento, que no genere desconciertos e incertidumbres, más bien la crisis del desarrollo que hoy día se vive a nivel planetario es porque el ser humano ha olvidado al hombre y a todas las dimensiones que lo integran. Las incertidumbres ocasionadas por las diversas corrientes ideológicas, políticas y filosóficas que han pretendido buscar solución al problema que genera el desarrollo, no han podido forjar una nueva conciencia en el pensamiento y en la acción, ya que las innovaciones para lograr el progreso surgen del inconsciente mismo del cuerpo social.

Morin (2002) insiste en que una nueva conciencia debería integrar: una reformulación del concepto de desarrollo, reestructurándolo para que éste no se encuentre subordinado al crecimiento; sino el crecimiento al desarrollo, evitando de esta forma las desesperadas maneras en que se pretende lograr el progreso.

El progreso y desarrollo entonces no pueden ser entendidos olvidando los aspectos fundamentales de la existencia humana, sino haciéndose cada vez más conscientes de aquellos procesos necesarios e importantes de todo crecimiento. La educación debe ser considerada como eje central y articulador de este proceso, integrando diversas formas para construir un conocimiento capaz de fortalecer el pensamiento y la acción. Según lo 
planteado por Castells (1997), la revolución experimentada en estos últimos años en el área educacional está dando paso a nuevos tipos de organización que se sustentan sobre la utilización cada vez más intensa del conocimiento y las tecnologías. De igual modo plantea que las personas, las ideas y los valores se están reestructurando de acuerdo a los avances vertiginosos de las nuevas tecnologías de la información, quienes están influyendo de manera considerable en los procesos que involucran el desarrollo de un país. La información, al igual que muchas otras áreas del desarrollo como lo es también la investigación, evidencia una profunda transformación, en sus vías de expansión, debido al avance de la ciencia y los eventos sociales que en los últimos años se han experimentado.

Estos cambios promueven el uso de nuevas tecnologías que facilitan la comunicación, pero al parecer los fines que movilizan y agitan las políticas nacionales empañan los aspectos equitativos que pudiera conllevar el acceso masivo a las fuentes informacionales, pudiendo formar personas más informadas, capaces de decidir y cuestionarse frente a las situaciones sociales, contrariando la visión reduccionista explicitada en el discurso sobre el ser humano, ampliando la noción de mano de obra calificada a un recurso mediante el cual se logren otros objetivos finales de mejora económica.

De esta forma, es importante considerar y comprender cuáles son las formas de concebir la información y mediante qué mecanismos logra generar cambios o permear la conducta de los individuos. Es por ello importante el aporte que presenta al proceso de desarrollo, en tanto posibilita y permite crear una plataforma mediante la cual se logra la construcción de significados, creando más allá de un desarrollo político o económico un desarrollo cultural. Esta transformación es la que, en definitiva, permitirá reformar las formas de convivir, trabajar y producir. Es en este nuevo paradigma cultural, en el cual debemos rescatar con prontitud los actos humanos que permitan centrar el crecimiento, progreso y desarrollo, en una visión mucho más compleja y multidimensional.

\subsection{NOCION DE CONOCIMIENTO}

Las principales proposiciones o atractores centrados en este aspecto se encuentran asociados a los conceptos avanzar en la ruta de la economía del conocimiento (concepto 4), utilización intensiva del conocimiento (concepto 12). Esto da cuenta de los modelos paradigmáticos que legitiman el proceso de obtención de conocimiento y lo configuran, permitiendo la aparición y expansión de emergentes formas de interpretación de los procesos cognitivos y sus implicancias en el desarrollo multidimensional de los países.

A pesar de que se reconoce la importancia del conocimiento en nuestro emergente escenario global, no es vista y analizada a la luz de diversas aristas, siendo más bien imperante las de orden político y económico. Sin embargo, el conocimiento no se puede situar únicamente bajo una arista unidimensional, entendiéndose como una correlación referida a los años de escolarización de una persona, tal como enfatizan los análisis de capital humano, ya que esto supone un reduccionismo y trivialización, que no aborda dominios esenciales del conocimiento, entre ellos la forma u organización paradigmática de determinadas instancias cognitivas y las redes comunicacionales emergentes productoras de esta forma u organización. (Oliva, 2007).

En el contexto de la denominada sociedad industrial, en que se vincula el conocimiento a un aspecto netamente económico, hablándose de economía del conocimiento, se observa 
como éste ha tendido a la reducción, en el paradigma de la simplicidad, reduciendo la complejidad mediante la disyunción, la unificación de lo múltiple, la eliminación del desorden y las contradicciones del conocimiento (Morin, Bocchi, Ceruti, 1991).

Aunque se insiste en que la nueva sociedad del conocimiento debiera permear las formas de gobierno y economía, es importante, a su vez, que las premisas sustentadas hasta hoy en la política de producción sean cambiadas, ya que se encuentran profundamente encarnadas e irrigan todos los aspectos de la dinámica social y cultural (Bateson, 1972). De allí la importancia que la esfera educativa, caracterizada por la idea de transmisión de unidades cognitivas, reinterprete la autonomía cognitiva y el carácter sistémico de los procesos de conocimiento. En este sentido von Foerster (1996:189), señala: "No es de extrañar que un sistema educacional que confunde el proceso de crear nuevos procesos (de distinciones) con la distribución de mercancías llamadas "conocimiento" puede causar alguna insatisfacción en los hipotéticos receptores, porque las mercancías no llegan: no hay tales mercancías".

\section{CONSIDERACIONES FINALES}

De acuerdo a los atractores obtenidos del plano argumental y el orden obtenido en términos de relaciones, la concepción de educación es considerada como central y neurálgica a lo largo del discurso, pero a pesar de que se reconoce su importancia y las instancias sobre las cuales se lleva a cabo dicho proceso, se imponen enfoques lineales y triviales de la cognición, unidimensionados en la competencia económica.

En ello se masifica y homogeniza la condición humana inhabilitando su capacidad de opinión e influencia en los eventos que comprenden su propia realización. Son otros los que evalúan su condición y los que, a la luz de sus propias certidumbres, en comparación a estándares que pueden disentir claramente de nuestra sociedad y cultura, deciden hacia dónde debemos ir y cuáles serán, a su entender, las mejoras que se obtendrán.

Queda implícito en la argumentación anterior que la relevancia del proceso educativo implica una concepción de la persona, de su constructo social, de las relaciones interpersonales, de la acción y de la ética. Si en el discurso público se desconocen estas instancias relacionales reduciendo el fenómeno a un mecanismo instrumental de "traspaso" de información o de la mera instrucción, convierte el proceso educativo en un mecanismo tecnocrático y deshumanizado, que en absoluto responde a las expectativas y necesidades de una sociedad compleja y diversa. Es por ello la importancia de una educación universal, integradora, flexible, humanística, que no sea mera acumulación tecnológica de datos (Franch, 1990)

La correspondencia lineal que se establece en el discurso de las instancias que se conciben necesarias para el desarrollo, como la productividad asociada a la mano de obra calificada, y la generación de recursos como meta fundamental, desestiman la importancia que presentan otras formas de desarrollo, asociadas a áreas que contemplen la diversidad y complejidad de los sistemas socioculturales. En este panorama, las concepciones de desarrollo, conocimiento y educación deben ser comprendidas en una dinámica y compleja red dialéctica, en la cual todos estos componentes sean considerados e integrados. 


\section{BIBLIOGRAFIA}

Bateson, G. (1972). "Steps To An Ecology Of Mind". Ballantine Books/Random House. New York.

Bernstein, B. (1993). "La estructura del discurso pedagógico". Madrid: Morata.

Brunner, J. (2000). "Nuevas Tecnologías y Sociedad de la Información". PREAL, Documento de trabajo 16. Santiago de Chile.

Carrizales, C. (1991). Las obsesiones pedagógicas de la modernidad. En C. Carrizales (Ed.), Modernidad y posmodernidad en educación. Cuernavaca: UAS y UAEM.

Castell, M. (1997). "La era de la información, Economía, Sociedad y Cultura: Fin de Milenio". Madrid: Alianza Editorial.

Chomsky, N. y H. Dieterich (1999). "La aldea global". Navarra: Txalaparta.

Coraggio, J. (1995). "Desarrollo Humano, Economía Popular y Educación”. Buenos Aires: AIQUEIDEAS.

Echeverría, R. (1994). "La Ontología del Lenguaje". Santiago de Chile: Dolmen Ediciones.

Fairclough, N. 1995. "Critical discourse analysis: The critical study of language". London y New York. Longman.

Ferrer, M. (1996). "La coherencia en el discurso coloquial". Rosario: Univ. Nacional de Rosario.

Foucault, M. (1983). "El discurso del poder". Ensayos y entrevistas compiladas y presentadas por Oscar Terán. México: Folios.

Franch, J. (1990). "Fundamentos del valor económico". Madrid: Unión Editorial.

Giddens, A. (1997). "Vivir en una sociedad postradicional", en Beck, U., Giddens, A. y Lash, S., Modernización reflexiva. Política, tradición y estética en el orden social moderno. Madrid, Alianza Universidad.

Lavanderos, L. y A. Malpartida (2000). "Tópicos en torno a la cognición relacional". Centro de Estudios en Teoría Relacional y Sistemas de Conocimiento. Santiago de Chile.

Martínez, M. (2001). "El contrato moral del profesorado. Condiciones para una nueva escuela". Bilbao: Desclée de Brouwer.

Morin, E., G. Bocchi y M. Ceruti (1991). "Un Nouveau Commencement”. Paris: Seuil.

Morin, E. y A. Kern. 1993. La reforma del pensamiento. En: Tierra Patria. Buenos Aires: Nueva Visión, 179-191 p.

Morin, E. (1999). "La cabeza bien puesta: Repensar la reforma $\leftrightarrow \rightarrow$ Reformar el pensamiento". Ed. Nueva Visión: Buenos Aires.

Morin, E. (2002). "La cabeza bien puesta: Repensar la reforma $\leftarrow \rightarrow$ Reformar el pensamiento". Ed. Nueva Visión: Buenos Aires.

Oliva, I. (2007). Aspectos Contextuales y Epistemológicos para una Transformación Educativa. Revista Complexus, Educación, Ciencia y Estética. Volumen 3, $\mathrm{N}^{\circ} 1$.

Torres, H. (1999). "Quiralidad en la Teoría de la Información aplicada al lenguaje genético". Revista Facultad de Ingeniería - Universidad de Tarapacá - Chile.

van Dijk, T. (1980). "Texto y Contexto". Madrid: Ediciones Cátedra.

van Dijk, T. (1983). "La ciencia del texto" Barcelona; Buenos Aires: Paidós.

van Dijk, T. (1885). "Handbook of discourse analysis". Academic press. London, New York: Toronto.

van Dijk, T. (2000) (ed). "Estudios del discurso" (2 vols.). Barcelona; Buenos Aires: Gedisa.

von Foerster, H. (1996). "La semilla de la cibernética". Edición de M. Pakman. Barcelona: Gedisa. 